\title{
Relating neural oscillations to laminar fMRI connectivity
}

\author{
René Scheeringa ${ }^{a, b, c, d^{*}}$, Mathilde Bonnefond ${ }^{c, e}$, Tim van Mourik $^{d}$, Ole Jensen ${ }^{f}$, David G. \\ Norris $^{\mathrm{a}, \mathrm{d}}$, Peter J. Koopmans ${ }^{\mathrm{a}, \mathrm{b}, \mathrm{d}}$ \\ ${ }^{a}$ University of Duisburg-Essen, Erwin L. Hahn Institute for Magnetic Resonance Imaging, UNESCO- \\ Weltkulturerbe Zollverein, Kokereiallee 7, 45141, Essen, Germany \\ ${ }^{b}$ University of Duisburg-Essen, University Hospital Essen, High-Field and Hybrid MR Imaging, Hufelandstr. 55, \\ 45147 Essen, Germany \\ ${ }^{c}$ Lyon Neuroscience Research Center, CRNL, Brain Dynamics and Cognition Team, INSERM U1028, CNRS \\ UMR5292, Lyon, France \\ ${ }^{d}$ Radboud University Nijmegen, Donders Institute for Brain, Cognition and Behaviour, Donders Centre for \\ Cognitive Neuroimaging, Trigon 204, P.O. Box 9101, 6500 HB Nijmegen, The Netherlands \\ e Université Claude Bernard Lyon 1, Université de Lyon, France
}

${ }^{f}$ School of Psychology, Centre for Human Brain Health, University of Birmingham, Hills Building, Birmingham B15 2TT, United Kingdom

\footnotetext{
*Corresponding author

Email:rene.scheeringa@donders.ru.nl
}

Keywords: laminar-fMRI, connectivity, EEG, neural oscillations 


\begin{abstract}
:
Laminar fMRI can non-invasively study brain activation and potentially connectivity at the laminar level in humans. In a previous simultaneous laminar fMRI/EEG experiment, we observed that attention effects in alpha, beta and gamma band EEG power relate to attention effects in fMRI activation in V1/V2/V3 at distinct cortical depths: alpha and gamma band EEG attention effects related to fMRI effects in superficial layers, whereas beta attention effects related to deep layers. Here we reanalyzed these data to investigate how EEG-attention effects relate to changes in connectivity between regions. We computed the fMRI-based attention effect on laminar connectivity between regions within a hemisphere and connectivity between layers within brain regions. We observed that the beta band strongly relates to laminar specific changes in connectivity. Our results indicate that the attentionrelated decrease in beta power relates to an increase in deep-to-deep layer connectivity between regions and deep/middle to superficial layer connectivity within brain regions. The attention related alpha power increase predominantly relates to increases in connectivity between deep and superficial layers within brain regions. We observed no strong relation between laminar connectivity and gamma band oscillations. These results indicate that especially beta band oscillations, and to a lesser extent alpha band oscillations relate to laminar specific changes in connectivity as measured by laminar fMRI. Together, the effects for the alpha and beta bands suggest a complex picture of possibly co-occurring neural processes that can differentially affect laminar connectivity.
\end{abstract}


bioRxiv preprint doi: https://doi.org/10.1101/2020.09.18.303263; this version posted September 18, 2020. The copyright holder for this preprint (which was not certified by peer review) is the author/funder, who has granted bioRxiv a license to display the preprint in perpetuity. It is made available under aCC-BY-NC 4.0 International license.

\section{Introduction}

Investigating directional laminar connectivity during task conditions has so far remained primarily the domain of invasive electrophysiological investigations in animals (1). In recent years, the development of high-resolution fMRI has made it possible to measure laminar level activity in humans noninvasively (2-9). Laminar specific anatomical connections directly relate to feedforward and feedback projections between regions at different levels in the cortical hierarchy $(10,11)$. These projections have been related to frequency-specific directional connectivity derived from electrophysiological measures. Recently, Sharoh et al.(9) demonstrated that laminar specific feedforward and feedback connections of the visual word form area with other regions can be studied with cortical depth resolved fMRI. Since frequency-specific electrophysiological synchronization has been directly related to laminar specific feedforward and feedback projections, this raises the question of how frequencyspecific neural synchronization relates to laminar level fMRI connectivity (12). Several frameworks for cortical processing explicitly link frequency-specific neural synchronization to laminar specific feedforward and feedback projections between brain regions (13-15). Laminar level fMRI connectivity could provide a unique opportunity to study such feedforward and feedback projections noninvasively in humans that can contribute to testing theoretical frameworks like these. For this, it is essential to first establish how neural oscillations in separate frequency bands relate to laminar connectivity. In an exploratory re-analysis of simultaneously recorded laminar fMRI-EEG data during an attention-demanding task (16) we therefore investigate how modulations if neural oscillations relate to changes in connectivity between regions in the early visual cortex.

Invasive recordings in animals have linked feedforward connectivity to inter-regional synchronization in the gamma and theta bands, while feedback has been linked to alpha and beta band synchronization $(1,13,17,18)$. Anatomically, these gamma/theta band feedforward connections are thought to originate from superficial (layers II/III) in lower order regions and target mainly middle layers (layer IV) in higher order regions. Feedback related alpha and beta band activity is thought to be conveyed from deep layers in higher order regions (layers V/VI) $(1,15,19)$ to layers outside layer IV in lower order regions, although for nearby regions feedback connections between superficial layers (layers II/III) have also been identified (20). Laminar fMRI allows us to study connectivity between various cortical depths non-invasively $(5,9,21,22)$ and when combined with electrophysiological measures like EEG this allows us to relate these effects to frequency specific neural synchronization. Here we implemented this combination by first computing individual task effects in cortical depth dependent correlation based connectivity. Subsequently we correlated this task effect over subjects with task effects in frequency specific EEG power bands. 
bioRxiv preprint doi: https://doi.org/10.1101/2020.09.18.303263; this version posted September 18, 2020. The copyright holder for this preprint (which was not certified by peer review) is the author/funder, who has granted bioRxiv a license to display the preprint in perpetuity. It is made available under aCC-BY-NC 4.0 International license.

In the original analyses of the data used here (16), we demonstrated that the alpha, beta and gamma band effects observed in this task related to the BOLD effect in different cortical layers. Alpha oscillations were found to correlate negatively with both deep and superficial layers, while beta oscillations were found to correlate negatively only with deep layer BOLD. For the gamma band a positive correlation was found in middle and superficial layers. These findings were largely in line with the likely laminar origins and function of these rhythms, suggesting a link between laminar BOLD and neural synchrony related feedforward and feedback projections between brain regions $(1,17)$. Crucially, the experimental paradigm task included a crude attention manipulation (attention ON vs. Attention OFF; see Fig. 1A) that forms the basis for the present contribution. We extend our previous work by correlating attention effects in laminar connectivity within and between regions in the visual cortex (V1, V2, V3), with attention effects in alpha, beta and gamma bands (see Fig. 1B-C for the original findings for this task contrast). These analyses will inform how specific frequency bands in EEG relate to cortical depth dependent fMRI connectivity between and within regions, and the results will be compared with their putative laminar specific roles in the feed-forward and feedback processes described above.

\section{$\underline{\text { Results }}$}

\section{Previous results}

A full account of our initial analyses and results can be found in Scheeringa et al. (16). In panel A of Fig. 1 we depict the structure of the experimental paradigm, an inward contracting circular sinusoidal grating, where subjects have to react to a speed increase in this contraction with a button press. Crucial for the analyses presented here, a cue indicated this was likely to occur ( $75 \%$ of the trials, $67 \%$ cue validity) or would not occur at all ( $25 \%$ of the trials, $100 \%$ cue-validity). The analysis presented here pertains to the two conditions where no button-press was required; the "attention ON" condition where a speed-change was cued but did not occur, and the "attention OFF" condition where no speed change was cued. This paradigm and task manipulation were chosen because they induce decreases in alpha and beta power of the EEG and increases in gamma band power relative to baseline, as well as in the attention ON condition compared to the attention OFF condition. This allows us to investigate the relationship of oscillatory power in different frequency ranges with laminar level BOLD activity (16) and connectivity (presented here) in the same task. As a consequence, our task modulation was not a clean modulation of attention and should not be interpreted as such, since relevant cognitive processes like arousal and the predictability of the speed change differed between the conditions. 
A

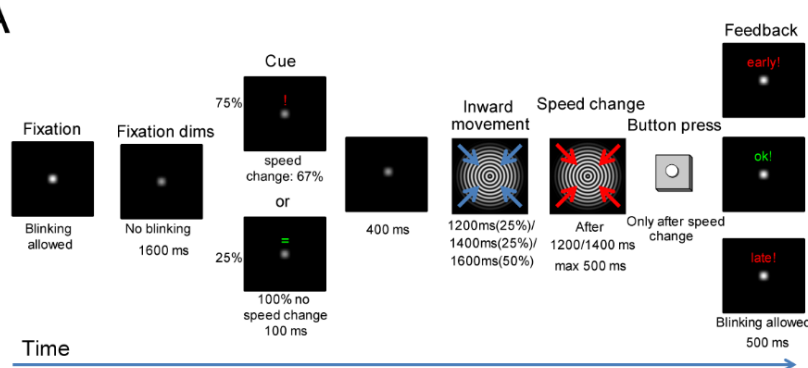

B

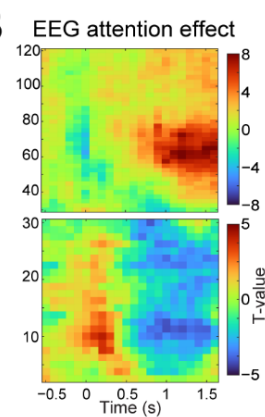

C FMRI attention effect

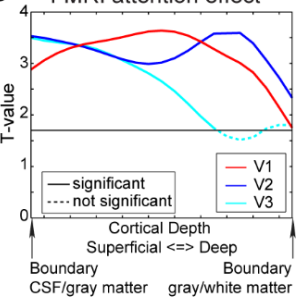

$\mathrm{D}$
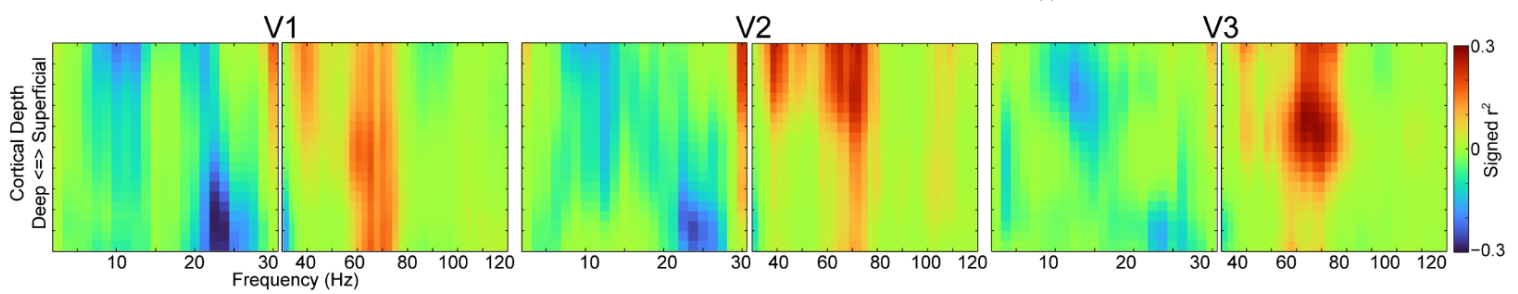

Fig. 1: Experimental paradigm and basic attention effects. A) Schematic representation of a trial of the experimental paradigm. B) Time-frequency of the attention effect on EEG power. C) Attention effect on depth resolved BOLD signal for V1, V2 and V3. D) Signed square correlation between the attention effect in the EEG power averaged over 0.6-1.6 s during the visual stimulation and the attention effect in depth resolved BOLD for V1, V2, V3. The results depicted are adapted from Scheeringa et al. (16).

In Panels 1B-D we depict the relevant results from this attention contrast taken from our previous work. In panel B we show a time-frequency representation of the effects on EEG power. It shows that alpha and beta power in the last $1 \mathrm{~s}$ of the trials is lower during "attention" on trials than during "attention OFF" trials, while gamma power is higher during the "attention ON" condition. In Panel C we show stronger BOLD activation during this condition across all cortical depths in V1, V2 and V3. For the $\mathrm{fMRI}$ signal we estimated the BOLD signal for 21 points between pairs of vertices at the CSF/gray matter and gray/white matter boundaries. The analyses presented here were carried out on BOLD data averaged from the top 10\% activated pairs of vertices in each region (collapsed over attention conditions and cortical depth). This selects the parts of the regions that were most active in response to the stimulus that spanned only a part of the visual field. Averaging over task activated regions in $\mathrm{V} 1 / \mathrm{V} 2 / \mathrm{V} 3$ is necessary to increase the signal to noise level, which is very low for the high-resolution individual voxels. The results for Panel $\mathrm{C}$ show the attention modulation leads to an increased BOLD response across all cortical depths in all three regions. In panel D we show the (signed squared) partial correlation between the attention effects in EEG power and those in laminar BOLD, while controlling for the effect of pupil diameter. These analyses show that the EEG alpha attention effect is negatively correlated with the BOLD attention effect in superficial layers for all regions, while the beta effect correlates negatively with the deep layer BOLD attention effect. For gamma we observed a positive correlation in middle/superficial depths for V2 and V3, and across all cortical depths for V1. 


\section{Laminar Connectivity}

A schematic representation of how we computed laminar connectivity and its relation to EEG power is depicted in Fig. 2. In panel A we illustrate how we compute the attention effect in laminar connectivity for a single combination of laminar depths of two regions (or similarly within a region), which was repeated for all region/depth combinations. We computed the attention effect in laminar connectivity in the following steps: First, we estimated the amplitude of single trial BOLD responses for the two conditions without a speed-increase (attention ON and attention OFF) by fitting a canonical hemodynamic response function. Subsequently, for each condition separately, the correlation of these amplitudes over trials (excluding erroneous trials and trials with eye blinks) was calculated. After applying a Fisher z-transformation to account for ceiling effects, these were averaged over the separate task blocks and subsequently grouped into inter-regional connectivity within a hemisphere, and intra-regional connectivity within the brain regions (see panel A of Fig. 3). Subsequently for each of the groups the attention contrast was calculated by subtracting the connectivity-metric for the "attention OFF" condition from the "attention ON" condition, the result of this analysis is illustrated in panel B. In order to relate EEG frequency bands to laminar fMRI connectivity, this attention contrast was then correlated over subjects (panel D) separately with the attention effects in respectively the alpha, beta and gamma bands (panel C) using a partial Spearman correlation (correcting for effects in the other frequency bands and pupil size). This results in a two dimensional representation of how laminar fMRI connectivity relates to a specific EEG frequency band, illustrated in panel E. The results of these analyses are depicted in panels B-D of Fig. 3. The previously observed attention effect in pupil size is depicted in Fig. S1, where we also show the correlation of the pupil-attention effect with laminar connectivity within and between regions, observing no significant effects. In addition, we also calculated the results for each region separately and their correlation with EEG are included in Fig. S2-S5). 

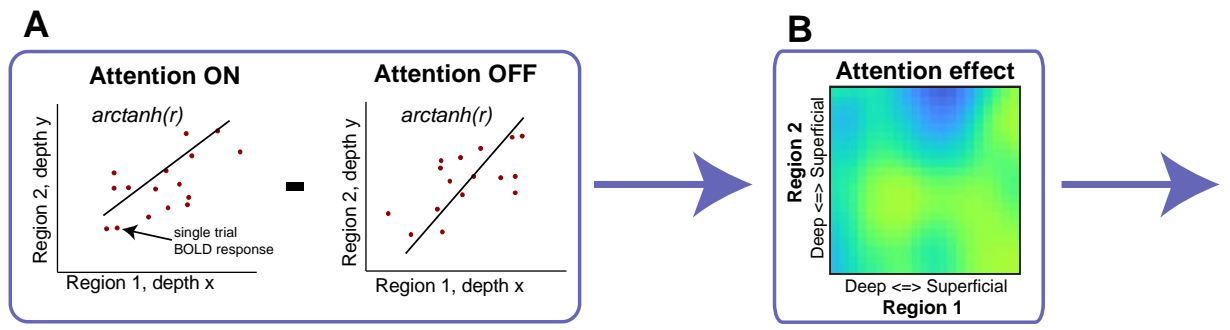

D
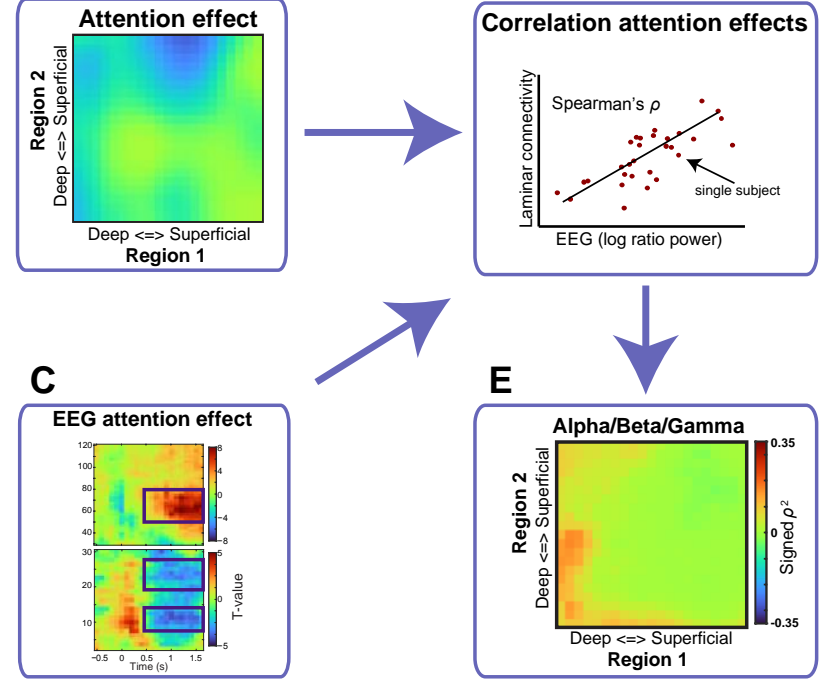

Fig. 2: Schematic representation of the analysis pipeline. Laminar connectivity was computed as the Fisher's z-transformation of the Pearson correlation ( $r$ ) between the amplitude of single trial BOLD responses, which were obtained through deconvolution (panels A) for both conditions and separately for each block. The attention effect for a single subject, depicted for each depth combination between regions in panel $B$, is computed as the difference between these $z$-transformed correlations averaged over the three experimental blocks. The single subject attention effects in the alpha, beta and gamma bands (panel C) were correlated over subjects with the single subject attention effects in laminar connectivity (panel D) using Spearman's rank-order correlation ( $\rho)$. After squaring and multiplication with the sign of the correlation, the laminar level relation of EEG frequency band with depth-resolved connectivity between region-pairs can be assessed separately for alpha, beta and gamma (panel E). For the analysis presented in the main article laminar connectivity was firs averaged over the region pairs indicated in Fig. 3 before the attention effect was calculated. For the results in Supporting Figs. S2, S3, S4 \& S5 this attention effect was calculated for individual region-pairs.

Fig. 3B depicts the average fMRI connectivity summed over the two conditions. For inter-region connectivity (top B), connectivity increased along the diagonal from deep-to-deep connectivity pairings to superficial-to-superficial pairings, in accordance with previous observations (22). Due to partial volume effects, connectivity is also strongest close to the diagonal for within region connections (on the diagonal it has by definition the value one). In panel $\mathrm{C}$ we depict the attention effects in BOLD connectivity. Between regions (top row), no strong laminar specific effects were observed, although there is a global trend that indicates a relative decrease with attention. Within 
brain regions (bottom row), a relatively strong, negative effect was found for connectivity between deep and superficial layers, as well as a similar non-specific decrease with attention.

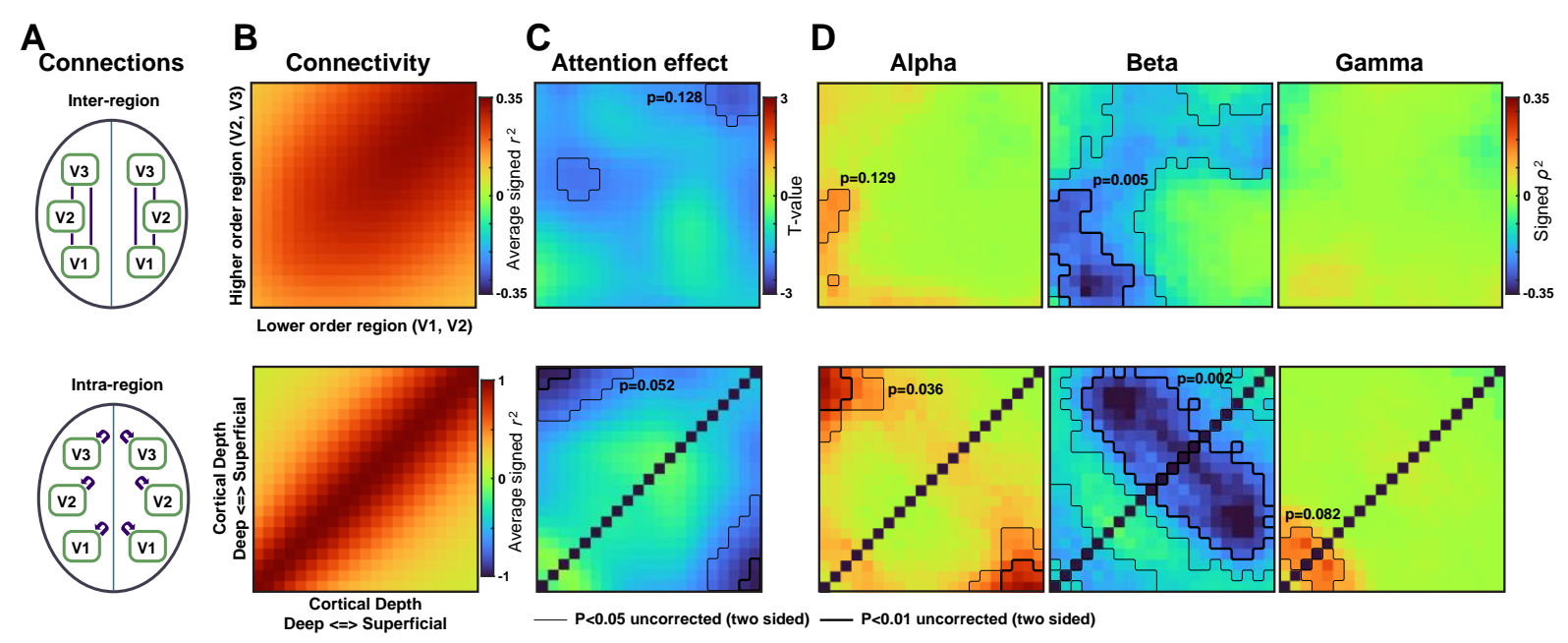

Fig. 3: Relation between EEG power and laminar fMRI connectivity. A) Schematic overview of the groupings of connections between and within regions (bottom schematic) over which the effects were averaged for the results depicted at the same rows in panels B-E. B) Average connectivity over attention ON and attention OFF conditions. C) Attention effect on laminar connectivity. D) Relation between attention effect in EEG power and laminar fMRI connectivity. Depicted is the signed squared partial Spearman correlation over subject $(n=30)$. For each frequency bands, the effects of other frequency bands and pupil size are partialed out. The $p$-values mentioned in panels $C$-D pertain to the largest supra-threshold cluster in each sub-panel and are based on a non-parametric cluster based permutation test. For all depictions in panels $B-D$, cortical depth combinations are similar as indicated for the attention effect in intra-regional connectivity in panel B (bottom).

In panel D of Fig. 3 we show how the attention contrast in laminar connectivity correlates with the attention effect in EEG power. The most prominent observation is that decreased beta band power strongly relates (over subjects) to increased laminar specific connectivity between regions within a hemisphere (top row) as well as within brain regions (bottom row). For laminar interactions between regions within a hemisphere, beta strongly correlates over subjects with deep-to-deep layer connectivity, while for connectivity within regions it relates to deep/middle to middle/superficial connectivity. The negative correlation with beta power is also observed for laminar connectivity between individual region pairs (Fig. S4). This indicates that the observed task related decrease in beta band power relates to both increased intra- and inter-regional laminar fMRI connectivity. For, the alpha band we observe a positive correlation with deep-to-superficial layer connectivity within a brain 
bioRxiv preprint doi: https://doi.org/10.1101/2020.09.18.303263; this version posted September 18, 2020. The copyright holder for this preprint (which was not certified by peer review) is the author/funder, who has granted bioRxiv a license to display the preprint in perpetuity. It is made available under aCC-BY-NC 4.0 International license.

region. Although there is no strong effect observed for alpha with between region connectivity, the trend seems to be the same, as well as for the relation of alpha with connectivity between single pairs of brain regions (see Fig. S3). Contrary to the beta effect, the attention related alpha power decrease seems therefore related to a decrease in connectivity. The relation of alpha power with deep-tosuperficial connectivity within brain regions shows a similar pattern to the attentional decrease in connectivity observed for these (c.f. Fig. $3 C$ and $D$ bottom row). To verify whether the effects observed for the alpha and beta bands are stable over analysis strategies, we repeated the analysis for the laminar signals extracted from the top 5\% (Fig. S6) and top 25\% (Fig. S7) activated signals. These analyses yielded largely similar results as those presented here.

\section{$\underline{\text { Discussion }}$}

In this study, we explore how task related changes in fMRI connectivity within and between brain regions relate to changes in neural synchronization measured by frequency specific EEG power. We used an attention demanding visual change detection task, which allowed us to correlate task induced differences in alpha, beta and gamma power to depth dependent connectivity between regions and within regions. We observed the strongest link between laminar fMRI connectivity and EEG power for the beta band. The negative correlation between beta power and deep-to-deep layer connectivity between regions and deep/middle to superficial layer connectivity indicates that a decrease in beta power reflects an increase in laminar specific connectivity. For the alpha band, we observed a positive correlation with deep-to-superficial layer connectivity within regions that resembled the inverse of the pattern of decreased connectivity that was also observed for the attention modulation. For connectivity between regions no significant relation was observed, although in general, a positive relation was present as well. This indicates that, in contrast to beta band decrease, the task induced alpha band decrease relates to decreased laminar connectivity, particularly within a region. For the gamma band, no relation was observed with laminar connectivity within regions, indicating that while gamma band activity is related to the strength of the BOLD signal in middle and superficial layers, this is not reflected in changes in laminar fMRI connectivity within and between brain regions.

The results for the beta band presented here support a notion that especially deep-to-deep layer feedback projections are related to beta band activity $(13,17,19)$. This is further supported by our previous finding from this dataset that beta power correlates negatively with BOLD activity in deep layers (16), which aligns well with observations in EEG and MEG that beta power, like alpha power, often decreases in regions that become actively involved in a task (23). On the other hand, inter-areal beta-band synchrony is regularly to increase when brain regions become task involved $(17,24,25)$. 
bioRxiv preprint doi: https://doi.org/10.1101/2020.09.18.303263; this version posted September 18, 2020. The copyright holder for this preprint (which was not certified by peer review) is the author/funder, who has granted bioRxiv a license to display the preprint in perpetuity. It is made available under aCC-BY-NC 4.0 International license.

This leads to a pattern of locally decreased beta band activity, reflected in a power decrease in EEG and MEG, and greater inter-regional beta band synchronization, which is typically better measured intracranially in animals. Here we find the deep-layer inter-regional connectivity increases for subjects that show a stronger task related beta power decrease. Following from the pattern described above, this increase might reflect the same underlying process that is reflected in (deep-to-deep layer) interregional beta band synchrony observed in animals. In addition, within a brain region we observed that a decrease in beta power predicts increased connectivity between deep/middle and superficial layers. Combined with the observation that beta correlates negatively with deep layer BOLD, and deep-todeep layer inter-regional connectivity, these findings indicate that within a brain region a decrease in beta power reflects increased incoming feedback related information in deep layers that is subsequently projected to middle/superficial layers.

For the alpha band, our main finding is that decreased alpha power (in the attention ON compared to the attention OFF condition) relates strongly to decreased correlations between deep and superficial layer BOLD-responses within the same region. This is in line with the observation in laminar recordings in monkeys that connected alpha sources can be found in both infragranular and supragranular layers (26-28). Typically, alpha is seen as either an idling rhythm that occurs when the cortex is not task involved, or a reflection of top-down inhibition of task irrelevant brain regions (29-31). The results here suggest these processes might be reflected in greater correlations between deep and superficial layer activity within regions. The inhibition or idling function of an increase alpha amplitude seems to be reflected in lower, but more correlated neural activity across layers. This increased correlation might reflect a lower capacity for independent and differentiated neural responses in different layers of the cortex, resembling observations task-evoked decreases in inter-neural correlations as measured by spike rate and fMRI connectivity (32-36). The results here suggest these decreases relate to taskevoked changes in local neural synchronization in the alpha band.

A striking negative finding here is the lack of a relation between gamma band activity and laminar connectivity. Gamma band activity is thought to reflect feed-forward flow of information from lower order superficial to higher order middle cortical layers $(13,15,17,18)$. In line with this, we found gamma band activity to correlate positively with middle/superficial layer BOLD responses to visual stimulation (16). We however did not find this reflected in a relation between gamma and laminar connectivity. Since electrophysiological measures of inter-regional connectivity have shown clear effects of attentional modulations $(18,37)$, this suggests that this type of neural synchronization between regions is not well captured by (laminar specific) hemodynamic connectivity measures. 
bioRxiv preprint doi: https://doi.org/10.1101/2020.09.18.303263; this version posted September 18, 2020. The copyright holder for this preprint (which was not certified by peer review) is the author/funder, who has granted bioRxiv a license to display the preprint in perpetuity. It is made available under aCC-BY-NC 4.0 International license.

Our observation that alpha and beta band activity have relationships with fMRI laminar connectivity that differ in sign (especially within a brain region) is surprising, since both bands show an attentional decrease in power and negatively correlate with the BOLD signal (Fig1D). Since alpha is thought to indicate functional inhibition or idling of task irrelevant regions (29-31), our results suggest increased BOLD connectivity, most pronounced for connectivity between layers within a region, can be a sign of decreased task involvement of connected but separate neural populations. Similarly as alpha, beta power is often observed to decrease in a context where regions become involved in task execution and increase when involvement stops, as is illustrated by the motor related beta-rebound $(38,39)$. However for beta, higher connectivity within and between regions is observed for subjects that showed a larger task related power decrease. Combined, these results for the alpha and beta bands indicate that an increase as well as a decrease in (laminar) fMRI connectivity measures can indicate greater involvement in the same task of separate neural populations (e.g. in different layers or regions). In general, this observation complicates the interpretation of $\mathrm{FMRI}$ based laminar and non-laminar connectivity on their own. Being able to relate (laminar) fMRI connectivity effects to EEG measures can facilitate this as is demonstrated here. However, a comparison of the pattern of attention effects in connectivity between regions in Fig. $2 \mathrm{C}$ with the observed relation with $\mathrm{EEG}$ frequency band in Fig. $2 D$, suggests that the pattern in attention effects cannot fully be explained by the EEG frequency bands under investigation here. This indicates that there are influences on task related fMRI connectivity changes that are not reflected in EEG power. Important in this respect is that fMRI is not able to measure neural activity like EEG and MEG. The latter critically depend on phase-synchronous responses in post-synaptic membrane potential of a part of the underlying neural population (apical dendrites of pyramidal cells), while fMRI can better be considered a representation temporally lowpass filtered total synaptic activity of the measured population.

The ability to study fMRI connectivity at the laminar level is a relatively recent development. Besides linking laminar the $\mathrm{fMRI}$ connectivity to frequency specific EEG power, the findings here also provide insight to laminar fMRI itself. A first pattern that emerges from our analysis is that irrespective of attention condition, we observed connectivity increased along the diagonal from deep-to-deep connectivity pairings to superficial-to-superficial pairings. This is in line with earlier observations of connectivity between voxels within a region (22), and is likely related to the larger contribution to the BOLD signal from draining veins which increases towards the surface of the cortex. When we subsequently compare the attention ON versus the attention OFF condition, the outcomes do suggest a general decrease in $\mathrm{fMRI}$ connectivity for both within and between region connectivity, Although no significant results were observed for our crude attention modulation in laminar fMRI connectivity within and between regions. While no particular laminar pattern stands out for connectivity between 
regions, connectivity between deep and superficial layers within a region was notably decreased. Since our task manipulation is not a clean modulation of attention but also includes cognitive processes like arousal and the predictability of the speed change, these decreases in connectivity cannot directly be linked to a specific cognitive process. It is however not uncommon to observe task-evoked decreases in neural correlations within a region and between connected cortical regions in both neural spiking and fMRI connectivity (32-36). Since our experimental manipulation essentially consisted of a task ON versus task OFF contrast, our results might reflect the same or a similar process. Our results suggest that this process of decorrelation of neural population, especially between different layers in the same region, is related to a decrease in alpha power.

In conclusion, this exploratory study provides the first evidence that neural oscillations measured by EEG reflect not only laminar specific neural activity, but also changes in laminar specific connectivity within and between brain regions. As such, it provides a neurophysiological basis for investigating laminar level connectivity with fMRI. It further suggests that the neural processes underlying alpha and beta oscillations are associated with opposite effects in correlation based connectivity measures in (laminar) fMRI, while their relations to the strength of the BOLD response and modulation by task conditions are highly similar. If this observation is indeed further verified, this could have important implications for correlation based measures of functional and effective connectivity. This would imply it is crucial to understand in which conditions alpha and beta neural oscillations are modulated in order to understand the effects of (laminar) connectivity changes in fMRI. Integrated analysis of laminar fMRI connectivity with electrophysiological measures like EEG and MEG might provide further insights on this topic.

\section{Methods}

The full preprocessing analysis pipeline for the data is described in our earlier work (16). Here we only repeat parts that are relevant for the additional analyses presented here.

\section{Subjects}

In this article we analyzed the data of the same thirty subjects that were included in the analysis of our earlier work ( 26 female, 4 male, age 18-26 years) out of originally 34 subjects ( 29 female, 5 male). These subjects participated in three blocks of an attention demanding visual task inside a 3T MRI scanner while simultaneously EEG, high resolution $\mathrm{fMRI}$, eye movements and pupil size was measured. 
bioRxiv preprint doi: https://doi.org/10.1101/2020.09.18.303263; this version posted September 18, 2020. The copyright holder for this preprint (which was not certified by peer review) is the author/funder, who has granted bioRxiv a license to display the preprint in perpetuity. It is made available under aCC-BY-NC 4.0 International license.

The experiment was approved by a local ethical committee (CMO Arnhem/Nijmegen region). For one of the included subjects only two blocks were carried out.

\section{Experimental paradigm and relevant previous results}

The details of the experimental paradigm and procedure are described in detail in Scheeringa et al. (16). A schematic overview is depicted in Fig1A. In short, subjects were required to fixate on a central fixation point, while simultaneously a circular inward contracting grating was presented for up-to 1600 ms. Their task was to respond with a button press as soon as the speed of the contraction increased. Crucial for the analyses presented here, a cue indicated whether this was likely to happen $(75 \%$ of the trials, $67 \%$ cue validity) or would not happen at all ( $25 \%$ of the trials, $100 \%$ cue-validity). The analysis presented here are pertain to the two conditions where no button-press was required; the "attention ON" condition where a speed-change was cued but did not occur, and the "attention OFF" condition where no speed change was cued. This task was chosen since it reliably induces decreases in alpha and beta power, and increases in gamma power. Except for one subject who stopped the measurement prematurely, all subjects performed three blocks of the task. Within each block there were 18 trials for both the attention ON condition without a speed change and the attention OFF condition.

After using an ICA denoising strategy (40), our previous analysis revealed indeed strong power decreases from baseline in alpha and beta bands, and increases in the gamma bands. Crucial for the analyses presented here, the last second of the trial, alpha and beta power was lower for the attention ON condition compared to the attention OFF condition while gamma power was higher in this condition (Fig. 1B. For the (laminar) depth resolved fMRI analysis, we first estimated the BOLD signal at 21 depths from the boundary between white and gray matter to the boundary between gray matter and CSF. Subsequently we averaged the BOLD signal for each bin over vertices within regions of interest in bilateral V1, V2, and V3. For the main analysis presented here we selected the top $10 \%$ activated vertices collapsed over cortical depth within these regions. Although arbitrary, a threshold is necessary to select the regions within V1, V2 and V3 that are involved in the task. For the three regions BOLD was observed to be higher in the attention ON condition across all cortical depths. Besides these attention effects in EEG power and BOLD, we also observed that the pupil size was larger during the last second of the trial during attention ON. In our previous analyses, we repeated the integrated EEG-fMRI analyses for the top $5 \%$ and $25 \%$ activated vertices to verify whether to the arbitrary choice of the threshold affected the results. For the analyses presented here we do this as well. 


\section{Estimating Laminar Connectivity}

In this study we estimate task related laminar fMRI connectivity from single trial BOLD amplitude estimates for all region/cortical depth combinations of the six regions involved. This was carried out by fitting the canonical HRF as implemented in SPM 12 (https://www.fil.ion.ucl.ac.uk/spm/) for every trial of the attention OFF and attention ON conditions without an increase in contraction speed. The other two conditions were each modeled by one regressor. The other included confound regressors were (i) two regressors modeling the button presses in the two trial types with a speed change; (ii) two regressors modeling the reaction time as a parametric modulation (one for each trial type with a speed change); (iii) regressors modeling the behaviorally incorrect trials for the two conditions with a speed change; (iv) the realignment parameters, their squares and derivatives to control for possible movement artifacts; $(v)$ a regressor modeling the T1 effect related to the pause after every third scan; and (vi) six sine and six cosine waves with frequencies up to $0.006 \mathrm{~Hz}$. We subsequently correlated for each condition the beta/parameter estimates of for every layer region/layer combination in every block for every subject. For the computation of this correlation, erroneous trials and trials including eye blinks were excluded. Subsequently, a Fisher-z transformation was applied to the correlations to account for the ceiling effects in correlation values. These values were then averaged over the task blocks and the region-combinations within each connectivity grouping as described in Fig $1 \mathrm{~A}$. The effect of the attention manipulation was calculated as the difference between the attention OFF minus attention $\mathrm{ON}$ estimate of laminar connectivity between these groupings. This attention effect was subsequently used to correlate over subjects with the attention effects in alpha, beta and gamma power and in pupil size. Significant attention effects in laminar connectivity were tested using a nonparametric cluster-based permutation test(41) implemented in Fieldtrip (42) for which the details are described in Scheeringa et al. (16). Clusters were defined by layer-by-layer combination for which $p<0.05$ uncorrected (two-tailed). We used 100000 permutations to estimate the reference distribution of cluster-sums.

\section{Correlating laminar connectivity with EEG power and pupil size}

We correlated the attention effects in the alpha $(7.5-12.5 \mathrm{~Hz})$, beta $(21.25-27.5 \mathrm{~Hz})$, and gamma (50$80 \mathrm{~Hz}$ ) bands as well as the attention effect we observed in pupil size with the attention effects in laminar connectivity for the four groupings. For these bands, we averaged over the attention effect, expressed as a log-ratio of the attention-on versus attention-off condition, over all time bins from 500 $\mathrm{ms}$ until 1,600 ms after the onset of visual stimulation and all frequency bins within the indicated 
ranges. For the pupil size, the log-ratio of the attention effect in pupil size was averaged over the same time-period. The partial Spearman correlation (assuming a monotonic, but not linear relationship), was calculated between the attention effects in laminar connectivity and the EEG frequency bands and pupil size. For each frequency in question, the effects in the other two frequency bands and pupil size were controlled for. For pupil size the correlation was controlled for the three EEG frequency bands. Significance was determined by a non-parametric cluster based test (41) applied to correlations that is described in full detail in our previous work(16). A cut-off of $p<0.05$ uncorrected (two-sided) was used to determine the clusters and 200000 permutations were used to calculate the reference distribution of cluster-sums.

\section{Acknowledgements}

This work was funded by the Emmy Noether Programme of the Deutsche Forschungsgemeinschaft (DFG), Grant KO 5341/1-1 to Peter Koopmans. René Scheeringa and Mathilde Bonnefond acknowledge support for the European Research Council under the European Union's Seventh Framework Programme (FP7/2007-2013)/ERC starting grant agreement no 716862. The data was recorded in a project funded by a grant from The Netherlands Organization for Scientific (NWO) to René Scheeringa (Veni scheme 451-12-021).

\section{$\underline{\text { References }}$}

1. van Kerkoerle T, et al. (2014) Alpha and gamma oscillations characterize feedback and feedforward processing in monkey visual cortex. Proceedings of the National Academy of Sciences of the United States of America 111(40):14332-14341.

2. Polimeni JR, Fischl B, Greve DN, \& Wald LL (2010) Laminar analysis of 7T BOLD using an imposed spatial activation pattern in human V1. Neurolmage 52(4):1334-1346.

3. Siero JC, Petridou N, Hoogduin H, Luijten PR, \& Ramsey NF (2011) Cortical depth-dependent temporal dynamics of the BOLD response in the human brain. $J$ Cereb Blood Flow Metab 31(10):1999-2008.

4. Muckli L, et al. (2015) Contextual Feedback to Superficial Layers of V1. Curr Biol 25(20):26902695.

5. Huber L, et al. (2017) High-Resolution CBV-fMRI Allows Mapping of Laminar Activity and Connectivity of Cortical Input and Output in Human M1. Neuron 96(6):1253-1263 e1257.

6. Finn ES, Huber L, Jangraw DC, Molfese PJ, \& Bandettini PA (2019) Layer-dependent activity in human prefrontal cortex during working memory. Nature neuroscience 22(10):1687-1695.

7. Lawrence SJ, Norris DG, \& de Lange FP (2019) Dissociable laminar profiles of concurrent bottom-up and top-down modulation in the human visual cortex. Elife 8.

8. Lawrence SJD, et al. (2018) Laminar Organization of Working Memory Signals in Human Visual Cortex. Curr Biol 28(21):3435-3440 e3434.

9. Sharoh $D$, et al. (2019) Laminar specific $\mathrm{fMRI}$ reveals directed interactions in distributed networks during language processing. Proceedings of the National Academy of Sciences of the United States of America 116(42):21185-21190. 
10. Douglas RJ \& Martin KA (2004) Neuronal circuits of the neocortex. Annu Rev Neurosci 27:419451.

11. Markov NT \& Kennedy H (2013) The importance of being hierarchical. Curr Opin Neurobiol 23(2):187-194.

12. Scheeringa R \& Fries $P$ (2017) Cortical layers, rhythms and BOLD signals. Neurolmage.

13. Bastos AM, et al. (2012) Canonical microcircuits for predictive coding. Neuron 76(4):695-711.

14. Bonnefond M, Kastner S, \& Jensen O (2017) Communication between Brain Areas Based on Nested Oscillations. eNeuro 4(2).

15. Fries P (2015) Rhythms for Cognition: Communication through Coherence. Neuron 88(1):220235.

16. Scheeringa R, Koopmans PJ, van Mourik T, Jensen O, \& Norris DG (2016) The relationship between oscillatory EEG activity and the laminar-specific BOLD signal. Proceedings of the National Academy of Sciences of the United States of America 113(24):6761-6766.

17. Bastos AM, et al. (2015) Visual Areas Exert Feedforward and Feedback Influences through Distinct Frequency Channels. Neuron 85(2):390-401.

18. Bosman CA, et al. (2012) Attentional stimulus selection through selective synchronization between monkey visual areas. Neuron 75(5):875-888.

19. Buffalo EA, Fries P, Landman R, Buschman TJ, \& Desimone R (2011) Laminar differences in gamma and alpha coherence in the ventral stream. Proceedings of the National Academy of Sciences of the United States of America 108(27):11262-11267.

20. Markov NT, et al. (2013) Cortical high-density counterstream architectures. Science 342(6158):1238406.

21. Huber L, et al. (2020) Layer-dependent functional connectivity methods. Prog Neurobiol:101835.

22. Wu PY, Chu YH, Lin JL, Kuo WJ, \& Lin FH (2018) Feature-dependent intrinsic functional connectivity across cortical depths in the human auditory cortex. Sci Rep 8(1):13287.

23. Spitzer B \& Haegens S (2017) Beyond the Status Quo: A Role for Beta Oscillations in Endogenous Content (Re)Activation. Eneuro 4(4).

24. Schoffelen JM, et al. (2017) Frequency-specific directed interactions in the human brain network for language. Proceedings of the National Academy of Sciences of the United States of America 114(30):8083-8088.

25. Buschman TJ \& Miller EK (2007) Top-down versus bottom-up control of attention in the prefrontal and posterior parietal cortices. Science 315(5820):1860-1862.

26. Bollimunta A, Chen Y, Schroeder CE, \& Ding M (2008) Neuronal mechanisms of cortical alpha oscillations in awake-behaving macaques. J Neurosci 28(40):9976-9988.

27. Bollimunta A, Mo J, Schroeder CE, \& Ding M (2011) Neuronal mechanisms and attentional modulation of corticothalamic alpha oscillations. J Neurosci 31(13):4935-4943.

28. Haegens S, et al. (2015) Laminar Profile and Physiology of the alpha Rhythm in Primary Visual, Auditory, and Somatosensory Regions of Neocortex. J Neurosci 35(42):14341-14352.

29. Jensen O \& Mazaheri A (2010) Shaping functional architecture by oscillatory alpha activity: gating by inhibition. Front Hum Neurosci 4:186.

30. Klimesch W, Sauseng P, \& Hanslmayr S (2007) EEG alpha oscillations: the inhibition-timing hypothesis. Brain Res Rev 53(1):63-88.

31. Pfurtscheller G, Stancak A, Jr., \& Neuper C (1996) Event-related synchronization (ERS) in the alpha band--an electrophysiological correlate of cortical idling: a review. Int J Psychophysiol 24(1-2):39-46.

32. Cohen MR \& Maunsell JH (2009) Attention improves performance primarily by reducing interneuronal correlations. Nature neuroscience 12(12):1594-1600.

33. Ito T, et al. (2020) Task-evoked activity quenches neural correlations and variability across cortical areas. PLoS Comput Biol 16(8):e1007983. 
34. Gonzalez-Castillo J \& Bandettini PA (2018) Task-based dynamic functional connectivity: Recent findings and open questions. Neurolmage 180(Pt B):526-533.

35. Ruff DA \& Cohen MR (2014) Attention can either increase or decrease spike count correlations in visual cortex. Nature neuroscience 17(11):1591-1597.

36. Cole MW, Bassett DS, Power JD, Braver TS, \& Petersen SE (2014) Intrinsic and task-evoked network architectures of the human brain. Neuron 83(1):238-251.

37. Grothe I, Neitzel SD, Mandon S, \& Kreiter AK (2012) Switching neuronal inputs by differential modulations of gamma-band phase-coherence. J Neurosci 32(46):16172-16180.

38. Pfurtscheller G, Stancak A, Jr., \& Neuper C (1996) Post-movement beta synchronization. A correlate of an idling motor area? Electroencephalogr Clin Neurophysiol 98(4):281-293.

39. Salmelin R, Hamalainen M, Kajola M, \& Hari R (1995) Functional segregation of movementrelated rhythmic activity in the human brain. Neurolmage 2(4):237-243.

40. Debener S, Ullsperger M, Siegel M, \& Engel AK (2006) Single-trial EEG-fMRI reveals the dynamics of cognitive function. Trends. Cogn. Sci. 10(12):558-563.

41. Maris E \& Oostenveld R (2007) Nonparametric statistical testing of EEG- and MEG-data. J Neurosci Methods 164(1):177-190.

42. Oostenveld R, Fries P, Maris E, \& Schoffelen JM (2011) FieldTrip: Open source software for advanced analysis of MEG, EEG, and invasive electrophysiological data. Comput Intell Neurosci 2011:156869. 